TRAVAIL

ET EMPLOI

\section{Travail et Emploi}

135 | juillet-septembre 2013

Expérimentations de terrain et politiques publiques du travail et de l'emploi. Apports récents et mises en perspective

\title{
Isabelle Bruno, Emmanuel Didier, Benchmarking. L'État sous pression statistique
}

Paris, Zones, 2013

Thomas Amossé

\section{(2) OpenEdition}

1 Journals

\section{Édition électronique}

URL : http://journals.openedition.org/travailemploi/6100

DOI : 10.4000/travailemploi.6100

ISSN : 1775-416X

Éditeur

DARES - Ministère du Travail

\section{Édition imprimée}

Date de publication : 15 septembre 2013

Pagination : 82-85

ISSN : 0224-4365

\section{Référence électronique}

Thomas Amossé, «Isabelle Bruno, Emmanuel Didier, Benchmarking. L'État sous pression statistique », Travail et Emploi [En ligne], 135 | juillet-septembre 2013, mis en ligne le 01 juillet 2013, consulté le 22 septembre 2020. URL : http://journals.openedition.org/travailemploi/6100 ; DOI : https://doi.org/ 10.4000/travailemploi.6100

Ce document a été généré automatiquement le 22 septembre 2020.

(C) Direction de l'animation de la recherche, des études et des statistiques (Dares) 


\section{Isabelle Bruno, Emmanuel Didier, Benchmarking. L'État sous pression statistique}

Paris, Zones, 2013

\section{Thomas Amossé}

\section{RÉFÉRENCE}

Isabelle Bruno, Emmanuel Didier, Benchmarking. L'État sous pression statistique, Paris, Zones, 2013, 209 p.

1 Cet ouvrage prend pour objet un phénomène à la présence diffuse et qui demeure pourtant assez largement mystérieux : le benchmarking. À partir de cet objet, il propose une grille de lecture stimulante des mutations actuelles de l'organisation du travail dans la fonction publique. Au ton militant mais à la rigueur scientifique indéniable, il dresse la sociogenèse de cette technique managériale née au début des années 1980 et présente une analyse critique de la technologie de gouvernement qu'elle est devenue. Respectivement politiste et sociologue, Isabelle Bruno et Emmanuel Didier proposent ainsi à la fois une histoire politique des outils de gestion des entreprises et une ethnographie des pratiques de travail dans l'administration. Ils mobilisent à cette fin des matériaux divers, comprenant des documents d'archives, des entretiens réalisés avec les concepteurs et utilisateurs de ces techniques et un large corpus de documents administratifs (extraits de rapports, tableaux de bord, etc.) qui donnent à voir le benchmarking en pratique. On notera la richesse particulière de l'enquête réalisée dans l'entreprise Xerox, là où est "née " la technique, dont les archives ont pu être confrontées au travers d'une série d'entretiens avec ce qu'en dit le "père officiel » (Robert Camp).

2 L'ouvrage est structuré en trois parties, qui comprennent une tentative de définition du benchmarking, un récit généalogique qui emmène le lecteur au Japon et aux États-Unis 
et une description de ses effets actuels en France sur trois terrains (la police, l'hôpital et l'université).

3 La définition que proposent les auteurs dans leur première partie n'en est pas à proprement parler une. Il s'agit d'une description technique de la forme que prend aujourd'hui dans l'administration «cet art bien spécifique de conduire les organisations sociales» (p.18). Partant du constat d'une «quantophrénie » dans la sphère publique, Isabelle Bruno et Emmanuel Didier identifient le benchmarking comme une des nouvelles (et principales) façons de quantifier l'activité des agents. La démarche comprend quatre étapes : la définition d'indicateurs ; la fixation d'objectifs à atteindre pour ces indicateurs; la définition d'une période de référence au terme de laquelle les objectifs doivent être remplis ; l'organisation, enfin, de lieux de rencontre où les acteurs se réunissent, se comparent et établissent de nouveaux objectifs. Suivant une sociologie de la quantification que n'aurait pas désavouée Alain Desrosières ${ }^{1}$, les auteurs détaillent les étapes de cette démarche et analysent les instruments quantitatifs sur lesquels elle s'appuie (tableaux de bord, palmarès et baromètre), qui relèvent d'une «nouvelle quantification publique » (p. 27). Référant l'esprit managérial qui prévaut aujourd'hui dans la fonction publique à ses fondements normatifs, ils tissent ensuite un premier fil qui relie les notions originelles du benchmarking ${ }^{2}$, du reengineering ${ }^{3}$ et du New Public Management ${ }^{4}$ à leur déclinaison actuelle dans l'administration française. Un dispositif, la LOLF (Loi organique relative aux lois de finance), symbolise cette déclinaison : comme l'indiquent Isabelle Bruno et Emmanuel Didier, «en généralisant la quantification de l'activité administrative tout en l'orientant dans le sens de la performance, elle a étendu et systématisé les réseaux du benchmarking au cœur de l'appareil étatique » (p. 60).

Plus que définitionnelle, la première partie pose les principaux éléments de la thèse qui, bien qu'implicite, soutient l'argumentation développée dans l'ouvrage : à savoir la continuité existant, d'un point de vue normatif et sous l'angle de l'instrumentation quantitative, entre le benchmarking originel, outil de gestion développé dans une entreprise américaine au tournant des années 1980, et le benchmarking d'aujourd'hui, principe d'organisation du travail dans la fonction publique. Les deuxième et troisième parties viendront étayer cette thèse en fournissant d'une part de nombreux indices de la circulation des discours et méthodes gestionnaires des États-Unis vers l'Europe et du monde de l'entreprise vers celui de l'administration, et en montrant, d'autre part, ce qu'est aujourd'hui le travail des agents publics dans des univers professionnels marqués par le déploiement de ce principe d'organisation.

5 La deuxième partie de l'ouvrage, de nature historique, est passionnante. Ce n'est pas une histoire, mais trois qui y sont retracées : celle des théories du contrôle du travail et du management de la qualité qui, en remontant aux années 1920, témoigne de l'allerretour des thématiques gestionnaires et organisationnelles entre les États-Unis et le Japon au cours du XXe siècle; celle ensuite du benchmarking qui a été développé dans l'entreprise Xerox, The Quality Company ${ }^{5}$, au tournant des années 1980, comme une des manières d'atteindre la "qualité totale "; celle, enfin, de l'importation dans le secteur public des méthodes de gestion venues du privé, qu'elles relèvent de la qualité, du benchmarking ou du reengineering. Ce dernier récit s'achève par la description de la naissance d'un benchmarking institutionnel portée par les instances européennes à compter de la deuxième moitié des années 1990 ( $c f$. la section «Cap sur l'entreprise Europe ", p. 114). 
6 La généalogie proposée, multiple nous l'avons écrit, se développe comme une toile d'araignée, avec de nombreux fils reliant différentes théories de gestion et d'organisation du travail, différents pays et secteurs d'activité. L'entreprise Xerox y occupe une place centrale, de même que certains de ses cadres tels que Camp (« inventeur » de la technique, dont les théories furent notamment reprises dans le milieu hospitalier, p. 157 et 158) ou David Kearns (président-directeur général de l'entreprise de 1982 à 1990, qui fut par la suite secrétaire adjoint à l'éducation du gouvernement fédéral où il conduisit une réforme d'ampleur visant à «appliquer à l'école ce qui avait marché chez Xerox sous la forme d'un "plan audacieux pour rendre les écoles compétitives dans la course mondiale des cerveaux" ", p. 111). Il en ressort une impression de porosité et en partie de continuité entre chacune des trois histoires. S'agissant de la naissance du benchmarking originel, le récit proposé invite à revenir sur le caractère pionnier de la méthode promue par Camp: son non competitive benchmarking (présenté p.94 et suivantes) étant de quelques années postérieur au competitive benchmarking de Charles Christ (pp. 92 -94).

7 La troisième partie s'attache à montrer de quelle manière le benchmarking qui s'est développé depuis une dizaine d'années dans l'administration en a transformé le travail. Trois terrains sont explorés, dans lesquels le lecteur ne peut que constater les effets délétères des politiques systématiques d'évaluation chiffrée de l'activité et de mise en concurrence, sur cette base, des services et des agents. L'enquête conduite dans la police prend sa source à New York, avec la mise en place de la politique du chiffre et du dispositif « Compstat » au milieu des années 1990. Le récit de son importation en France au début des années 2000, dont témoignent de nombreux acteurs clés (dont le préfet de police de Paris), est édifiant: il donne à voir le déploiement d'une organisation administrative visant à évaluer, comparer et récompenser (ou pénaliser) l'ensemble des commissariats, organisation dont les conséquences les plus sensibles ont pu être observées en bas de la hiérarchie policière. On découvre l'état de surrégime de cette administration et les stratégies de résistance, comme la «chanstique " qui consiste à jouer avec les règles de report des statistiques (p. 143). Suit la description de la situation de l'hôpital, qui remonte pour la France au début des années 1980 avec la mise en place du programme de médicalisation des systèmes d'information (PMSI), programme qui attribue à chaque unité de soin un ensemble de points en fonction des actes médicaux réalisés. La logique de comparaison des établissements ne vient que bien plus tard, en 1998, par le biais d'un article de journal ayant publié un palmarès des hôpitaux construit à partir des données du PMSI. Elle est relayée à l'échelon politique à partir du début des années 2000 et est systématisée à l'occasion des différentes réformes hospitalières mises en œuvre depuis. Pour l'enseignement supérieur et la recherche, le développement du benchmarking suit le même trajet que dans la police ou pour l'hôpital, des États-Unis vers la France, ou plus largement l'Europe, puisque cette méthode a de fait été directement intégrée à la politique européenne à partir de la déclaration de Bologne (1999). Elle a pesé dans la «révolution en cours de l'université française ", suscitant une large vague de protestation.

8 L'ouvrage d'Isabelle Bruno et Emmanuel Didier est passionnant, nous l'avons écrit. Les réflexions qui y sont proposées sont stimulantes et les enquêtes sur lesquelles il s'appuie sont riches. Sa lecture n'emporte toutefois pas totalement l'adhésion, ce qui renvoie peut-être à l'impossible définition de son objet et à la thèse qui lui sert de fondement. L'unité postulée du phénomène que constitue le benchmarking, la continuité 
qui relierait sa version originelle à celle qui est actuellement un des principes des réformes à l'œuvre dans la fonction publique n'a rien d'évident. Elle semble même s'effacer dès lors que l'on relie, non plus pas à pas, mais d'une extrémité à l'autre le parcours généalogique que propose l'ouvrage. Qu'y a-t-il de commun entre la veille des meilleures pratiques ouverte sur l'ensemble de l'économie (promue par Camp) et la politique organisée du chiffre dans la police qui s'est développée quinze ans plus tard ?

Il nous semble qu'il y aurait un grand avantage d'un point de vue analytique à relire cette histoire, non plus en pensant le commun, mais en soulignant les glissements d'une période à l'autre, les différences d'un pays ou d'un univers professionnel à l'autre. Il ne s'agit bien sûr pas de gommer les évidents liens de filiation qui existent entre les acceptions originelles et les déclinaisons plus récentes du benchmarking, qui ne sont pas que terminologiques. Mais une fois levée la contrainte de devoir convaincre et être convaincus qu'un seul et même phénomène existe à trente ans d'écart, des entreprises états-uniennes aux commissariats parisiens, auteurs et lecteurs seraient me semble-t-il mieux à même de comprendre la transformation de cet outil de gestion en technologie de gouvernement. La circulation des techniques de management pourrait être mieux objectivée. Cela permettrait d'éviter l'illusion de la nouveauté ${ }^{6}$ et de cerner ce qui est spécifique à chaque forme de benchmarking (les deux premiers développés chez Xerox, ses déclinaisons dans le reengineering, etc.). C'est d'une importance cruciale sur un point, celle de la place des statistiques, qui n'est que secondaire pour Camp (p. 44) ou Hammer et Champy (p. 48). Ainsi pourrait-on mieux cerner la place et le rôle des instruments quantitatifs dans les différentes formes de benchmarking.

10 En conclusion, les auteurs annoncent un second ouvrage autour de la notion de statactivism, qui vise à rendre compte du potentiel subversif des outils de quantification. On ne peut que s'en réjouir. Une autre piste pourrait être suivie, plus ambitieuse, qui viserait à donner plus d'ampleur aux analyses proposées ici : il s'agirait d'établir une histoire politique qui relierait l'origine du contrôle du travail au début du $\mathrm{XX}^{\mathrm{e}}$ siècle à la réforme de l'État cent ans plus tard, en lien avec les théories et méthodes de gestion, et les formes, statistiques ou non, d'évaluation du travail. Les riches matériaux collectés nous semblent pouvoir constituer un premier socle empirique à cette fin. Certains sujets, qui ne sont qu'esquissés ici pourraient aussi être développés, comme le lien entre l'avènement du benchmarking actuel dans la fonction publique et l'évolution des inégalités au travail en son sein. De façon plus fondamentale, une théorie de l'État pourrait être proposée puisqu'une transformation plus importante que celle décrite par Karl Polanyi ${ }^{7}$ est annoncée dans l'ouvrage (p. 11).

11 Quoi qu'il en soit, en attendant de nouvelles parutions d'Isabelle Bruno et Emmanuel Didier, on ne peut que recommander la lecture de cet ouvrage qui avance, par ses résultats comme ses hypothèses, nombre de réflexions utiles pour comprendre l'évolution récente des organisations du travail dans l'administration et des formes de contrôle qui s'y exercent. 


\section{NOTES}

1. Paru peu de temps après son décès, l'ouvrage lui est dédié.

2. Selon Robert Camp, lu par Isabelle Bruno et Emmanuel Didier, cette notion peut être "définie » comme une systématisation en entreprise de " comparaisons sans cesse renouvelées [qui] doivent permettre d'identifier la "meilleure des meilleures" façons de faire, dont on s'inspirera en vue d'une "amélioration continue" de ses propres procédés » (p. 41). Pour plus de précision, cf. Camp R. (1989), Benchmarking. The search for industry best practices that lead to superior performance, Milwaukee, ASQC Quality Press.

3. Le reengineering se situe dans le prolongement du benchmark, qu'il entend dépasser: il est présenté comme "une révolution du monde des affaires", susceptible de "réinventer l'entreprise pour une amélioration spectaculaire de ses performances » en "cré[ant] le nouveau benchmark de classe mondiale " (cf. Hammer M., Champy J. [1993], Reengineering the corporation. A manifesto for business revolution, New York, Harper Business, cités page 46 par Isabelle Bruno et Emmanuel Didier).

4. Soulignant que "l'acronyme générique NPM ne saurait épuiser en trois initiales l'extrême variété des pratiques»(p.49), les auteurs relèvent l'importance de l'ouvrage Reinventing governement. How the entrepreneurial spirit is transforming the public sector (David Osborne et Ted Gaebler, Reading, Addison Wesley, 1992), présenté comme un «livre manifeste [...] [où sont définis] un nouveau paradigme gouvernemental, une nouvelle façon de conduire les organisations, publiques comme privées » (pp. 49-50).

5. Selon son slogan publicitaire dans les années 1980 (p. 89).

6. Le benchmarking a quelque parenté avec des pratiques plus anciennes, comme la notation des agents (évoquée p.17), le stakhanovisme (p.25) et des formes d'évaluation comparative des performances existant en République démocratique allemande (RDA) dans les années 1980 (p. 65). Aussi, aimerait-on que soit davantage discuté ce qu'il y a de spécifiquement nouveau dans ce phénomène.

7. Polanyi K. (1983), La grande transformation. Aux origines politiques et économiques de notre temps, Paris, Gallimard.

\section{AUTEURS}

\section{THOMAS AMOSSÉ}

Centre d'études de l'emploi (CEE) 